\title{
Hemogasometria e variáveis cardiopulmonares após administração do butorfanol em cães anestesiados pelo desfluorano sob ventilação espontânea
}

\author{
Acid-base and cardiopulmonary effects after butorphanol administration in spontaneously breathing \\ dogs anesthetized by desflurane
}

\author{
Paulo Sérgio Patto dos Santos ${ }^{{ }^{*}}$ Newton Nunes ${ }^{\mathrm{II}}$ Almir Pereira de Souza ${ }^{\mathrm{III}}$ \\ Márlis Langenegger de Rezende ${ }^{\mathrm{IV}}$ Celina Tie Nishimori ${ }^{\mathrm{IV}}$ \\ Danielli Parrilha de Paula ${ }^{\mathrm{IV}}$ Piedad Natalia Henao Guerrero ${ }^{\mathrm{IV}}$
}

\section{RESUMO}

Este experimento teve por objetivos avaliar as possíveis alterações cardiopulmonares e hemogasométricas decorrentes do uso do butorfanol em cães submetidos à anestesia pelo desfluorano sob ventilação espontânea. Para tal, foram utilizados vinte cães adultos, clinicamente saudáveis, pesando $12 \pm 3 \mathrm{~kg}$. Os animais foram distribuídos igualmente em dois grupos, GS e GB, e induzidos à anestesia com propofol $\left(8,4 \pm 0,8 \mathrm{mg} \mathrm{kg}^{-1}, \mathrm{IV}\right)$, intubados e submetidos à anestesia inalatória pelo desfluorano (10V\%). Decorridos 40 minutos da indução, foi administrado aos animais do GS $0,05 \mathrm{~mL} \mathrm{~kg}$ de solução fisiológica a $0,9 \%$ (salina), enquanto que, no $G B$, foi aplicado butorfanol na dose de $0,4 \mathrm{mg} \mathrm{kg}^{-1}$, ambos pela via intramuscular. As observações das variáveis freqüências cardíaca (FC) e respiratória (f), pressões arteriais sistólica $(P A S)$, diastólica (PAD) e média (PAM), pH arterial $(p H)$, pressão parcial de oxigênio no sangue arterial $\left(\mathrm{PaO}_{2}\right)$, pressão parcial de dióxido de carbono no sangue arterial $\left(\mathrm{PaCO}_{2}\right)$, déficit de base $(\mathrm{DB})$, bicarbonato $\left(\mathrm{HCO}_{3}\right)$ e saturação de oxigênio na hemoglobina $\left(\mathrm{SatO}_{2}\right)$ tiveram início imediatamente antes da aplicação do opióide ou salina (M0). Novas mensurações foram realizadas 15 minutos após a administração do butorfanol ou salina (M15) e as demais colheitas foram realizadas a intervalos de 15 minutos, por um período de 60 minutos (M30, M45, M60 e M75). Os dados numéricos dessas variáveis foram submetidos à Análise de Perfil $(P<0,05)$. A freqüencia cardíaca apresentou alteração no GB, com média de MO maior que as demais. As PAS, PAD e PAM, assim como a $f$ e o $\mathrm{pH}$, apresentaram valores menores após a administração do opióide no $G B$, em comparação ao GS. A $\mathrm{PaO}_{2}$ apresentou discretas alterações, entretanto sem significado clínico, enquanto que a $\mathrm{PaCO}_{2}$ e $\mathrm{o} \quad \mathrm{DB}$ apresentaram valores de M0 menores que os demais após a aplicação do butorfanol. Os resultados obtidos permitem concluir que a administração do butorfanol em cães submetidos à anestesia pelo desfluorano, sob ventilação espontânea, reduz a freqüencia cardíaca e a pressão arterial e promove certo grau de hipoventilação.

Palavras-chave: butorfanol, cães, hemogasometria, desfluorano.

\section{ABSTRACT}

The cardiopulmonary and acid-base effects of butorphanol in desflurane anesthetized dogs breathing spontaneously were evaluated. Twenty adult healthy, male and female dogs were used. They were separated into two groups of 10 animals each (GS and GB). Anesthesia was induced with propofol $\left(8.4 \pm 0.8 \mathrm{mg} \mathrm{kg}^{-1} \mathrm{IV}\right)$ and maintained with desflurane (10V\%). After 40 minutes of induction, the animals from GS received saline solution at $0.9 \%\left(0.05 \mathrm{~mL} \mathrm{~kg}^{-1}\right)$ and from $\mathrm{GB}$ received butorphanol $\left(0.4 \mathrm{mg} \mathrm{kg}^{-1}\right)$, both applied intramuscularly. Heart (HR) and respiratory (RR) rates; systolic (SAP), diastolic $(D A P)$ and mean (MAP) arterial pressures; arterial blood $\mathrm{pH}$ $(\mathrm{pH})$, arterial partial pressure of $\mathrm{O}_{2}\left(\mathrm{PaO}_{2}\right)$ and arterial partial pressure of $\mathrm{CO}_{2}\left(\mathrm{PaCO}_{2}\right)$; base deficit $(\mathrm{BD})$, arterial oxygen saturation ( $\left.\mathrm{SaO}_{2}\right)$ and bicarbonate ion concentration $\left(\mathrm{HCO}_{3}\right)$ were measured. The measurements were taken immediately before the application of the agents (M0). Serial measurements were carried out at 15 minutes intervals after the administration of butorphanol or saline up to 75 minutes (M15, M30, M45, M60 and M75). Data were submitted to Profile Analysis $(P<0.05)$. After butorphanol administration HR, SAP, DAP and MAP decreased significantly. $\mathrm{PaO}_{2}$ had discreet alterations, however without clinical meaning. $R R$ and $p H$ decreased after butorphanol administration while $\mathrm{PaCO}_{2}$ increased significantly. It was possible to conclude that butorphanol administration in desflurane anesthetized dogs produced reduction in the averages of heart rate and arterial pressure and relatively to the respiratory parameters, the opioid produced hypoventilation in spontaneously breathing dogs.

Key words: butorphanol, desflurane, dogs, blood gases.

ICurso de Medicina Veterinária, Centro Universitário Barão de Mauá (CBM), 14031-580, Ribeirão Preto, SP, Brasil. E-mail: pspatto@itelefonica.com.br.*Autor para correspondência.

IIDepartamento de Clínica e Cirurgia Veterinária, Faculdade de Ciências Agrárias e Veterinárias (FCAV), Universidade Estadual Paulista (UNESP), Jaboticabal, SP, Brasil.

"IUniversidade Federal de Campina Grande (UFCG), Campus de Patos, Patos, PB, Brasil.

IVPrograma de Pós-graduação em Cirurgia Veterinária, FCAV, UNESP, Jaboticabal, SP, Brasil. 


\section{INTRODUÇÃO}

Ao longo dos anos, tem-se buscado a atualização de técnicas e conceitos que tornem a anestesia segura e aplicável aos vários procedimentos cirúrgicos que se fazem necessários à prática da Medicina Veterinária. Dentre estes, técnicas multimodais que associam agentes voláteis ou intravenosos com opióides ou anestesia regional têm sido empregadas, com o objetivo de se obter efeitos sinérgicos desejáveis inerentes aos diversos fármacos e técnicas. Nesse contexto, o uso de agentes analgésicos se destaca, uma vez que é crescente a preocupação com o bem-estar animal e com a redução na quantidade do anestésico geral normalmente empregada, de modo a propiciar recuperação anestésica segura e suave.

Quanto aos fármacos analgésicos, os opióides se sobressaem, pois sua eficácia e segurança os tornam indicados para o tratamento da dor em diversas circunstâncias. Dentre os opióides, o butorfanol apresenta propriedades vantajosas, uma vez que a ação mista resultante de seu efeito agonista e antagonista proporciona sedação e analgesia, com menor depressão cardiorrespiratória, quando comparado a outros opióides (FANTONI \& MASTROCINQUE, 2002). O butorfanol atua no nível subcortical do sistema límbico (BOOTH \& McDONALD, 1992), sendo considerado 4 a 7 vezes mais potente que a morfina (THURMON et al., 1996).

O uso isolado do butorfanol proporciona mínimas alterações nos sistemas cardiovascular e respiratório (QUANDT et al., 1994; SCRIVANI et al., 1998); entretanto, quando empregado associado ao midazolam, foram observadas significativa redução de freqüência respiratória e elevação da concentração de dióxido de carbono no ar expirado $\left(\mathrm{ETCO}_{2}\right)$ e da pressão arterial de dióxido de carbono $\left(\mathrm{PaCO}_{2}\right)$ (GROSS et al., 1993). A depressão respiratória causada pelo fármaco é menor que a proporcionada pela morfina e os efeitos depressores são limitantes (TRIM, 1983; THURMON et al., 1996; FANTONI \& MASTROCINQUE, 2002). Por outro lado, WATERMAN et al. (1991) não observaram alterações hemogasométricas quando da administração de butorfanol na dose de $0,2 \mathrm{mg} \cdot \mathrm{kg}^{-1}$ em ovelhas, enquanto que TYNER et al. (1989) observaram elevação na $\mathrm{PaCO}_{2}$ e redução do pH arterial em cães, como resposta à diminuição da amplitude e da freqüência respiratória.

Sobre a atividade cardiovascular em cães, o butorfanol reduz a freqüência cardíaca, a pressão arterial (TRIM, 1983; GREENE et al.,1990; QUANDT et al.,1994) e o débito cardíaco (TYNER et al.,1989). Adose normalmente empregada em cães é de 0,2 a $0,4 m g$ kg$^{-1}$ e pode-se administrar o butorfanol por via intravenosa, intramuscular e subcutânea. Apresenta período de latência ao redor de 16 minutos e sua duração de ação é em torno de 4 horas em cães (FANTONI \& MASTROCINQUE, 2002).

O desfluorano, introduzido na prática clínico-cirúrgica em 1992, apresenta baixo coeficiente de solubilidade sangue/gás $(0,42)$, tornando rápidas a indução e a recuperação do paciente (EGER, 1992). A administração do agente halogenado produz aumento da atividade simpática, a qual atinge o pico máximo decorridos 5 minutos de exposição ao fármaco (PACENTINE et al., 1995).

No sistema cardiovascular, atua diminuindo, de modo dependente da dose, o débito cardíaco (PAGEL et al., 1998), a pressão arterial (CIOFOLO \& REIZ, 1999) e a resistência vascular sistêmica (LOWE et al., 1996; RODIG et al., 1997), enquanto promove aumento da freqüência cardíaca (CLARKE et al., 1996). O desfluorano apresentou efeitos cardiorrespiratórios similares aos induzidos pelo isofluorano antes, durante e após ventilação seletiva em pacientes humanos submetidos a cirurgia pulmonar (PAGEL et al., 1998). Sobre a função respiratória, diversos estudos em animais sob ventilação espontânea demonstraram que o agente produz depressão de modo dependente da dose (CLARKE, 1999; SANTOS, 2000). Quando associado ao óxido nitroso, pôde-se observar depressão respiratória e esse achado aponta o desfluorano como agente depressor respiratório mais potente que o gás (NISHIMORI, 2003).

Dessa forma, com o intuito de proporcionar informações complementares ao profissional de Anestesiologia Veterinária, relativas a efeitos ainda pouco elucidados, objetivou-se estudar os efeitos hemogasométricos e cardiovasculares advindos da administração do butorfanol em cães durante anestesia pelo desfluorano (1,4 CAM), sob ventilação espontânea.

\section{MATERIAL E MÉTODOS}

Foram utilizados 20 cães adultos, machos e fêmeas, sem raça definida, pesando $12 \pm 3 \mathrm{~kg}$, considerados sadios após avaliação clínica e laboratorial, fornecidos pelo canil do Hospital Veterinário “Governador Laudo Natel”, da FCAV/ UNESP, Campus de Jaboticabal, SP. Após serem selecionados aleatoriamente, os animais foram distribuídos em dois grupos de igual número $(n=10)$, denominados grupo salina (GS) e grupo butorfanol (GB).

Os animais foram induzidos à anestesia geral pela administração intravenosa de propofol ${ }^{\mathrm{a}}$ na dose 
de $8,4 \pm 0,8 \mathrm{mg} \mathrm{kg}^{-1}$. Os cães, após colocados em decúbito dorsal sobre colchão térmico ativo, foram intubados com sonda de Magill e submetidos à anestesia inalatória pelo desfluorano ${ }^{\mathrm{b}}$, administrado diluído em $\mathrm{O}_{2}\left(30 \mathrm{~mL} \mathrm{~kg}^{-1} \mathrm{~min}^{-1}\right)$, por meio de circuito anestésico com reinalação parcial de gases ${ }^{c}$, dotado de vaporizador $^{\mathrm{d}}$ termocompensado, microprocessado e calibrado para o agente anestésico. O desfluorano foi fornecido na concentração de 1,4 CAM (10V\%), considerando-se uma CAM como sendo equivalente a 7,2V\% (CLARKE et al., 1996), mensurada em equipamento digitale ${ }^{\text {e }}$ cujo sensor foi adaptado na extremidade da sonda orotraqueal e conectado ao circuito anestésico. Após 40 minutos da indução anestésica, foram administrados aos animais do GS $0,05 \mathrm{~mL} . \mathrm{kg}^{-1}$ de solução fisiológica a $0,9 \%$ (salina), enquanto, nos do GB, foi aplicado butorfanol ${ }^{\mathrm{f}}$ na dose de $0,4 \mathrm{mg} \mathrm{kg}^{-1}$, ambos pela via intramuscular e em volume final iguais.

A freqüência cardíaca (FC) foi obtida por meio de eletrocardiógrafo computadorizado ${ }^{g}$, ajustado para leitura na derivação DII. A determinação das pressões arteriais sistólica (PAS), diastólica (PAD) e média (PAM) foi feita por leitura direta, em monitor multiparamétrico ${ }^{\text {h }}$, cujo transdutor foi conectado em cateter introduzido na artéria femoral esquerda. A freqüência respiratória $(f)$ foi obtida por leitura

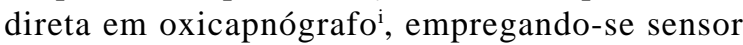
de fluxo principal conectado entre a sonda orotraqueal e o equipamento de anestesia. As variáveis hemogasométricas pressão parcial de $\mathrm{O}_{2}$ no sangue arterial $\left(\mathrm{PaO}_{2}\right)$; pressão parcial de $\mathrm{CO}_{2}$ no sangue arterial $\left(\mathrm{PaCO}_{2}\right)$; saturação de oxihemoglobina no sangue arterial $\left(\mathrm{SatO}_{2}\right)$; déficit de base (DB); bicarbonato $\left(\mathrm{HCO}_{3}\right)$ e $\mathrm{pH}$ do sangue arterial foram obtidas empregando-se equipamento específico ${ }^{j}$, por meio de colheita de amostra de sangue, no volume de 0,3mL, colhida através do cateter empregado na mensuração das pressões arteriais.

As observações das variáveis estudadas tiveram início imediatamente antes da aplicação do opióide ou solução de cloreto de sódio a 0,9\% (M0). As demais colheitas e registros dos dados foram realizadas em intervalos de 15 minutos, por um período de 60 minutos, M30, M45, M60 e M75, respectivamente. A avaliação estatística foi efetuada por meio de Análise de Perfil (MORRISON, 1967 e CURI, 1980), considerando um nível de significância de $5 \%(\mathrm{P}<0,05)$.

\section{RESULTADOS}

A FC não apresentou alteração das médias entre os grupos, assim como no grupo GS, enquanto que, no GB, a média de M0 foi maior que as demais. Os valores relativos às pressões arteriais, na comparação entre grupos, mostrou que, em M15, M30, M45, M60 e M75, o GB apresentou valor médio menor que o do GS. No estudo dos grupos isoladamente, observou-se que, no GB, as médias foram menores após o emprego do butorfanol (Tabela 1).

Quanto a $f$, na comparação entre os grupos, observou-se que o GB apresentou valores médios menores que no GS de M15 a M75. Já a análise isolada dos grupos mostrou que no GB a média observada em M30 foi menor que a de M0. A análise da $\mathrm{PaCO}_{2}$ revelou que, de M30 a M75, o GB apresentou valores médios maiores que os do GS, e a média observada em M0 foi menor que às de M30 a M75 no GB, enquanto que em M75 o GB apresentou $\mathrm{PaO}_{2}$ menor que no GS (Tabela 2). As médias do DB não apresentaram alterações entre os grupos. Na análise dos grupos individualmente no GB, as médias de M45 a M75 foram maiores que a de M0 e a média de M45 foi maior que as de M15 e M30. Já quanto ao $\mathrm{pH}$, a comparação entre os grupos revelou que de M30 a M75, o GB apresentou valores menores que os de GS. Para as variáveis $\mathrm{SaO}_{2}$ e $\mathrm{HCO}_{3}$, não houve diferenças entre grupos nem entre os momentos em ambos os grupos (Tabela 2).

\section{DISCUSSÃO}

Com relação à metodologia empregada, esta foi delineada visando a avaliar as alterações promovidas pelo butorfanol durante anestesia com o desfluorano. Dessa forma optou-se por administrar o opióide com os animais já em plano anestésico, como fora empregado por TYNER et al. (1989) e GREENE et al. (1990). Assim, pôde-se obter resultados que efetivamente demonstraram a ação do opióide durante a anestesia com desfluorano.

Para a indução anestésica, optou-se pelo emprego do propofol. A dose empregada foi de $8,4 \pm 0,8 \mathrm{mg} \mathrm{kg}^{-1}$, com a qual foi possível obter bons planos anestésicos sem excitação ou efeitos adversos, permitindo a intubação adequada de todos os animais. Relativamente ao momento estabelecido para o início da colheita dos dados, optou-se por iniciá-lo 40 minutos após a indução anestésica (M0), tempo requerido para a cateterização da artéria femoral e suficiente para a completa exclusão dos possíveis efeitos decorrentes da administração do propofol (ZORAN et al., 1993).

Dessa forma e com base nos resultados obtidos, pode-se tecer algumas considerações relativas à administração de butorfanol em cães anestesiados pelo desfluorano. Nesse sentido, o desfluorano não alterou a freqüência cardíaca, como fora citado por CLARKE et al. (1996), permanecendo numa faixa 
Tabela 1 - Valores médios e desvios padrão $(\mathrm{x} \pm \mathrm{s})$ das variáveis: freqüência cardíaca (FC), pressão arterial sistólica (PAS), diastólica (PAD) e média (PAM) obtidos em cães anestesiados pelo desfluorano (1,4 CAM), sob ventilação espontânea e tratados com salina (GS) ou butorfanol (GB).

\begin{tabular}{|c|c|c|c|c|c|c|c|}
\hline Variáveis & & M0 & M15 & M30 & M45 & M60 & M75 \\
\hline \multirow{2}{*}{ FC (batimentos.min..$^{-1}$ ) } & GS & $133 \pm 17$ & $131 \pm 17$ & $131 \pm 18$ & $130 \pm 18$ & $130 \pm 17$ & $128 \pm 19$ \\
\hline & GB & $138 \pm 21 \mathrm{a}$ & $118 \pm 20 b$ & $119 \pm 20 b$ & $121 \pm 20 b$ & $124 \pm 21 b$ & $124 \pm 22 b$ \\
\hline \multirow{2}{*}{ PAS (mmHg) } & GS & $115 \pm 16$ & $116 \pm 14$ & $115 \pm 14$ & $115 \pm 14$ & $115 \pm 12$ & $113 \pm 11$ \\
\hline & GB & $115 \pm 11 \mathrm{a}$ & $91 \pm 12 b *$ & $93 \pm 9 b^{*}$ & $93 \pm 7 b^{*}$ & $94 \pm 7 b *$ & $94 \pm 8 b *$ \\
\hline \multirow{2}{*}{ PAD (mmHg) } & GS & $63 \pm 13$ & $65 \pm 15$ & $64 \pm 14$ & $62 \pm 14$ & $61 \pm 13$ & $60 \pm 12$ \\
\hline & GB & $67 \pm 10 \mathrm{a}$ & $47 \pm 8 b^{*}$ & $47 \pm 8 b^{*}$ & $47 \pm 9 b^{*}$ & $47 \pm 12 b *$ & $46 \pm 11 b^{*}$ \\
\hline \multirow{2}{*}{ PAM (mmHg) } & GS & $83 \pm 11$ & $85 \pm 12$ & $84 \pm 13$ & $83 \pm 12$ & $82 \pm 11$ & $81 \pm 10$ \\
\hline & GB & $86 \pm 10 a$ & $64 \pm 10 b^{*}$ & $65 \pm 8 b^{*}$ & $65 \pm 8 b^{*}$ & $66 \pm 10 b^{*}$ & $65 \pm 9 b *$ \\
\hline
\end{tabular}

*Existe diferença estatística entre os grupos para este momento pela Análise de Perfil $(\mathrm{P}<0,05)$.

Médias seguidas por letras diferentes, nas linhas, diferem significativamente entre si pela Análise de Perfil $(\mathrm{P}<0,05)$.

considerada adequada à espécie. Por outro lado, a associação com butorfanol induziu efeito cronotrópico negativo, diminuindo a freqüência cardíaca 15 minutos após a administração do opióide, permanecendo neste patamar por todo o período experimental, conforme observado por GREENE et al. (1990), embora, neste caso, a FC tenha permanecido reduzida somente 30 minutos após a administração do opióide. Tal evento está relacionado ao aumento da atividade vagal (GREENE et al., 1990; QUANDT et al., 1994), embora este efeito seja mais pronunciado quando da administração intravenosa de butorfanol.

Relativamente às pressões arteriais, a literatura consultada cita que o desfluorano promove redução da PAM, na medida em que as concentrações anestésicas são aumentadas (LEUNG \& PASTOR, 1998; CIOFOLO \& REIZ, 1999). Todavia, esse parâmetro permaneceu estável ao longo de todo o período experimental, uma vez que não houve alteração das concentrações inspiradas do agente volátil. Entretanto, a adição do butorfanol causou diminuição das pressões arteriais, o que, de acordo com TYNER et al. (1989), pode ser atribuído à redução da resistência periférica total (RPT) promovida pelo agente devido à ação do fármaco no sistema nervoso central, ou diretamente sobre a musculatura lisa dos vasos (WHITE et al., 1990). Ainda quanto às pressões arteriais, pode ter ocorrido efeito aditivo do desfluorano aos do opióide sobre a musculatura vascular, facilitando o relaxamento e propiciando esta diminuição de pressão (RODIG et al., 1997).

Quanto à freqüência respiratória, observouse redução da variável imediatamente após a administração do butorfanol, permanecendo reduzida por todo o período experimental. Este achado pode ser decorrente da associação do agente volátil com o opióide, de modo a produzir maior depressão respiratória, uma vez que os opióides são depressores do sistema respiratório (THURMON et al., 1996), embora alguns autores reportarem que o butorfanol produz menor depressão respiratória quando comparado com outros opióides (TRIM, 1983; THURMON et al., 1996; FANTONI \& MASTROCINQUE, 2002).

Já quanto ao desfluorano, diversos estudos em animais sob ventilação espontânea demostraram que o agente produz depressão respiratória de modo dependente da dose (CLARKE, 1999; SANTOS, 2000). Todavia, as mudanças relativas à freqüência respiratória no grupo que recebeu placebo não foram significativas, o que permite inferir que o agente volátil proporciona estabilidade respiratória em concentrações fixas, normalmente empregadas na rotina clínica e cirúrgica. Entretanto, é importante salientar que a análise da $f$, isoladamente, não é representativa do perfil respiratório, sendo portanto necessário estudos relacionados aos efeitos da associação de desfluorano e butorfanol, em cães, sobre a ventilometria ou em doses distintas dos fármacos de modo a confirmar ou não esta hipótese.

A $\mathrm{PaCO}_{2}$ comportou-se de modo inversamente proporcional à $f$, visto que relacionam-se intimamente. Estatisticamente, foram encontradas diferenças entre os grupos 30 minutos após o emprego do butorfanol, quando foi possível observar aumento do parâmetro, reforçando a afirmação de depressão do sistema respiratório, decorrente da administração do 
Tabela 2 - Valores médios e desvios-padrão $(\mathrm{x} \pm \mathrm{s})$ das variáveis: freqüência respiratória $(f)$, pressão parcial de $\mathrm{CO}_{2}$ no sangue arterial $\left(\mathrm{PaCO}_{2}\right)$, pressão parcial de $\mathrm{O}_{2}$ no sangue arterial $\left(\mathrm{PaO}_{2}\right)$, saturação de oxihemoglobina no sangue arterial $\left(\right.$ Sat $\left.\mathrm{O}_{2}\right)$, déficit de base (DB), bicarbonato $\left(\mathrm{HCO}_{3}\right)$ e $\mathrm{pH}$ do sangue arterial obtidos em cães anestesiados pelo desfluorano (1,4 CAM), sob ventilação espontânea e tratados com salina (GS) ou butorfanol (GB).

\begin{tabular}{|c|c|c|c|c|c|c|c|}
\hline Variáveis & & M0 & M15 & M30 & M45 & M60 & M75 \\
\hline \multirow{2}{*}{$f\left(\right.$ mov.min. $\left.^{-1}\right)$} & GS & $13 \pm 6$ & $15 \pm 6$ & $15 \pm 6$ & $16 \pm 6$ & $15 \pm 5$ & $16 \pm 8$ \\
\hline & GB & $11 \pm 2 \mathrm{a}$ & $9 \pm 3 a b^{*}$ & $8 \pm 3 b^{*}$ & $9 \pm 3 \mathrm{ab}^{*}$ & $9 \pm 3 a b^{*}$ & $9 \pm 3 a b^{*}$ \\
\hline \multirow{2}{*}{$\mathrm{PaCO}_{2}$ (mmHg) } & GS & $48,9 \pm 6$ & $49,8 \pm 8$ & $48,2 \pm 5$ & $46,8 \pm 6$ & $47,0 \pm 5$ & $48,4 \pm 7$ \\
\hline & GB & $50,0 \pm 5 \mathrm{a}$ & $53,6 \pm 7 \mathrm{ab}$ & $56,1 \pm 7 b^{*}$ & $56,5 \pm 6 b^{*}$ & $57,5 \pm 6 b^{*}$ & $58,6 \pm 6 b^{*}$ \\
\hline \multirow{2}{*}{$\mathrm{PaO}_{2}(\mathrm{mmHg})$} & GS & $455 \pm 36$ & $474 \pm 31$ & $482 \pm 34$ & $469 \pm 49$ & $486 \pm 42$ & $497 \pm 31$ \\
\hline & GB & $461 \pm 46$ & $445 \pm 33$ & $477 \pm 32$ & $474 \pm 41$ & $475 \pm 33$ & $450 \pm 46^{*}$ \\
\hline \multirow{2}{*}{$\mathrm{SaO}_{2}(\%)$} & GS & $100 \pm 0$ & $100 \pm 0$ & $100 \pm 0$ & $100 \pm 0$ & $100 \pm 0$ & $100 \pm 0$ \\
\hline & GB & $100 \pm 0$ & $100 \pm 0$ & $100 \pm 0$ & $100 \pm 0$ & $100 \pm 0$ & $100 \pm 0$ \\
\hline \multirow{2}{*}{ DB (mEq. $\left.\mathrm{L}^{-1}\right)$} & GS & $-2,5 \pm 3$ & $-2,0 \pm 3$ & $-2,2 \pm 3$ & $-2,4 \pm 3$ & $-2,2 \pm 3$ & $-2,3 \pm 3$ \\
\hline & GB & $-3,2 \pm 3$ a & $-2,7 \pm 3$ & $-2,3 \pm 3$ & $-1,3 \pm 3 b$ & $-1,7 \pm 3 b$ & $-1,5 \pm 3 b$ \\
\hline \multirow{2}{*}{$\mathrm{HCO}_{3}\left(\mathrm{mEq} \cdot \mathrm{L}^{-1}\right)$} & GS & $24 \pm 2$ & $24 \pm 3$ & $24 \pm 3$ & $24 \pm 2$ & $24 \pm 2$ & $24 \pm 3$ \\
\hline & GB & $23 \pm 3$ & $24 \pm 3$ & $24 \pm 3$ & $25 \pm 4$ & $25 \pm 3$ & $25 \pm 3$ \\
\hline \multirow{2}{*}{$\mathrm{pH}$} & GS & $7,30 \pm 0,05$ & $7,30 \pm 0,05$ & $7,31 \pm 0,04$ & $7,31 \pm 0,04$ & $7,32 \pm 0,04$ & $7,30 \pm 0,05$ \\
\hline & GB & $7,28 \pm 0,05$ & $7,27 \pm 0,06$ & $7,26 \pm 0,05^{*}$ & $7,26 \pm 0,05 *$ & $7,25 \pm 0,05^{*}$ & $7,25 \pm 0,05^{*}$ \\
\hline
\end{tabular}

* Existe diferença estatística entre os grupos para este momento pela Análise de Perfil $(\mathrm{P}<0,05)$.

Médias seguidas por letras diferentes, nas linhas, diferem significativamente entre si pela Análise de Perfil $(\mathrm{P}<0,05)$.

opióide (THURMON et al., 1996; FANTONI \& MASTROCINQUE, 2002). O incremento nos valores médios obtidos pode ser conseqüência da supressão da resposta do centro respiratório a $\mathrm{PaCO}_{2}$, a qual pode resultar em hipercapnia, atribuída a redução da amplitude e da freqüência respiratórias (CORNICK \& HARTSFIELD, 1992). O fato de o butorfanol ter produzido elevação significativa da $\mathrm{PaCO}_{2}$ somente 30 minutos após a sua administração justifica-se, uma vez que os eventos que acometem a dinâmica respiratória não produzem respostas imediatas, sendo, portanto, necessário algum tempo para que a elevação da pressão parcial de $\mathrm{CO}_{2}$ sangüínea, decorrente da redução da $f$, possa ser detectada pela hemogasometria.

Entretanto, é importante destacar que, embora tenha ocorrido elevação da $\mathrm{PaCO}_{2}$, os achados são condizentes com certo grau de hipoventilação e não de hipoxemia, visto que os valores obtidos de $\mathrm{PaO}_{2}$ e da $\mathrm{SatO}_{2}$ foram suficientemente elevados para garantir o suprimento de oxigênio para os tecidos e as médias de $\mathrm{PaCO}_{2}$ mantiveram-se discretamente acima dos valores considerados normais para a espécie, adotandose como parâmetro cães submetidos à anestesia pelo halotano (HASKINS, 1996).
Resultados semelhantes também foram obtidos por MCMURPHY \& HODGSON (1996), segundo os quais a pressão parcial arterial de $\mathrm{CO}_{2}$ foi maior em gatos anestesiados pelo desfluorano a 1,7 do que a 1,3 CAM, durante respiração espontânea, demonstrando novamente a interferência da concentração anestésica sobre essa variável. Por outro lado, deve-se salientar que os valores médios obtidos em M0, em ambos os grupos, apresentaram-se discretamente acima dos valores considerados normais, demostrando que o desfluorano promove alteração na $\mathrm{PaCO}_{2}$, produzindo médias elevadas, provavelmente devido a sua ação depressora sobre o sistema respiratório (CLARKE, 1999; SANTOS, 2000) e ao concomitante aumento do shunt pulmonar promovido pelo agente volátil (NUNES et al., 2003).

Quanto à saturação de oxihemoglobina no sangue arterial $\left(\mathrm{SatO}_{2}\right)$, embora efetivamente não represente a quantidade de oxigênio disponível para os diversos tecidos, esta permite determinar a oxigenação arterial e pode ser empregada como indicativo de distúrbios que possam representar situação de risco para os animais, principalmente durante procedimentos cirúrgicos. Nesse sentido, os 
achados relativos à variável, em ambos os grupos, não apresentaram quaisquer alterações significativas, mantendo as médias estáveis e dentro da faixa de normalidade para a espécie (HASKINS, 1996).

Relativamente ao déficit de base (DB), não foram observadas diferenças entre os grupos, embora deva-se considerar que os elevados coeficientes de variação possam reduzir a sensibilidade do teste estatístico. Por outro lado, os animais que receberam butorfanol apresentaram elevação significativa da variável 45 minutos após a administração do opióide, o que demonstra alguma compensação metabólica, com retenção de bicarbonato, sem contudo caracterizar acidose metabólica, uma vez que os valores referentes ao DB mantiveram-se dentro da faixa de normalidade para a espécie (LUNA, 2002). A variação do déficit de base pode ser explicada como sendo consequiência da redução da $f$ e da subseqüente elevação da $\mathrm{PaCO}_{2}$, perfil este freqüentemente observado com o emprego de opióides (THURMON et al., 1996; FANTONI \& MASTROCINQUE, 2002).

Ainda quanto às variáveis estudadas, é de extrema relevância tecer considerações relativas à concentração anestésica do desfluorano empregada. A literatura consultada é unânime ao afirmar que os opióides proporcionam redução significativa da concentração alveolar mínima (CAM) dos diversos agentes halogenados (VALVERDE et al., 1989; SEBEL et al., 1992; KATOH et al., 1999; NUNES et al., 2001). Dessa forma, a concentração empregada neste estudo não foi eqüipotente, uma vez que não se procedeu a sua redução após a administração do butorfanol. Frente a isso, devido ao menor requerimento anestésico nesses animais e à depressão atribuída ao agente volátil ser dependente da dose, pode-se esperar que os dados obtidos dos animais que receberam o opióide tenham sido afetados.

\section{CONCLUSÕES}

De acordo com os resultados obtidos com a metodologia empregada, pode-se concluir que a administração do butorfanol em cães anestesiados com desfluorano reduz a freqüência cardíaca e as pressões arteriais e pode ser empregado com segurança, pois não promove alterações cardiorrespiratórias importantes apesar, de induzir certo grau de hipoventilação.

\section{AGRADECIMENTOS}

À Fundação de Amparo à Pesquisa do Estado de São Paulo-FAPESP, pelo apoio financeiro concedido.

\section{FONTES DE AQUISIÇÃO}

aDIPRIVAN - ZENECA Farmacêutica do Brasil Ltda. - São Paulo, SP, Brasil.

'bUPRANE - ZENECA Farmacêutica do Brasil Ltda. - São Paulo, SP, Brasil.

'OHMEDA - mod. Excel 210SE - Datex Ohmeda - Miami, EUA. (Processo FAPESP 97/10668-4).

dOHMEDA - mod. TEC 6 - Datex Ohmeda - Miami, EUA. (Processo FAPESP 97/10668-4).

'OHMEDA - mod. RGM 5220 - Datex Ohmeda - Miami, EUA. (Processo FAPESP 96/12830-0).

'TORBUGESIC - Fort Dodge Saúde Animal Ltda. - Campinas, SP, Brasil.

gTEB - mod. ECGPC software versão 1.10 - São Paulo, SP, Brasil. (Processo FAPESP 96/1151-5).

hDixtal - mod. DX2010 - PA invasiva - Manaus, AM, Brasil. (Processo FAPESP 96/02877-0).

'Dixtal mod. DX 7100 - Manaus, AM, Brasil. (Processo FAPESP 97/10668-4).

iI-Stat - SENSOR DEVICES, INC. - Nova Jersey, EUA (Processo FAPESP 96/12830-0).

\section{COMISSÃO DE ÉTICA}

Trabalho de pesquisa aprovado pela Comissão de Ética e Bem-Estar Animal da FCAV/Unesp Jaboticabal, protocolo $n^{\circ}$ 003724-05.

\section{REFERÊNCIAS}

BOOTH, N.H.; McDONALD, L.E. Farmacologia e terapêutica em veterinária. 6.ed. Rio de Janeiro: Guanabara Koogan, 1992. p.234-267.

CIOFOLO, M.J.; REIZ, S. Circulatory effects of volatile anesthetic agents. Minerva Anestesiol, Turin, v.65, n.5, p.232-238, 1999.

CLARKE, K.W. et al. Cardiopulmonary effects of desflurane in the dog during spontaneous and artificial ventilation. Research in Veterinary Science, London, v.61, p.82-86, 1996.

CLARKE, K.W. Desflurane and sevoflurane: new volatile anesthetic agents. Veterinary Clinics of North America: Small Animal Practice, Philadelphia, v.29, n.3, p.793-810, 1999.

CORNICK, J.L.; HARTSFIELD, S.M. Cardiopulmonary and behavioral effects of combinations of acepromazine/butorphanol and acepromazine/oxymorphone in dogs. Journal of the American Veterinary Medical Association, Schaumburg, v.200, n.12, p.1952-1956, 1992.

CURI, P.R. Análise de medidas repetidas em experimentos biológicos. Revista Brasileira de Estatística, São Paulo, v.41, n.161, p.137-150, 1980.

EGER, E.I. Desflurane animal and human pharmacology: aspects of kinetics, safety, and MAC. Anesthesia and Analgesia, New York, v.75, p.3-9, 1992.

FANTONI, D.T.; MASTROCINQUE, S. Fisiopatologia e controle da dor. In: FANTONI, D.T.; CORTOPASSI, S.R. Anestesia em cães e gatos. São Paulo: Roca, 2002. p.323-336. 
GREENE, S.A. et al. Cardiovascular effects of butorphanol in halothane-anesthetized dogs. American Journal of Veterinary Research, Schaumburg, v.51, n.8, p.1276 -1279, 1990.

GROSS, M.E. et al. Cardiorespiratory effects of combined midazolam and butorphanol in isoflurane-anesthetized cats. Veterinary Surgery, Philadelphia, v.22, n.2, p.159-162, 1993.

HASKINS, S.C. Monitoring the anesthetized patient. In: THURMON, J.C. et al. Lumb \& Jones' veterinary anesthesia. 3.ed. Philadelphia: Lea \& Feabiger, 1996. p.418.

KATOH, T. et al. The effect of fentanyl on sevoflurane requirements for somatic and sympathetic responses to surgical incision. Anesthesiology, Hagerstown, v.90, n.2, p.398-405, 1999.

LEUNG, J.M.; PASTOR, D.A. Dissociation between haemodynamics and sympathetic activation during anaesthetic induction with desflurane. Canadian Journal of Anaesthesia, Ontário, v.45, n.6, p.533-540, 1998.

LOCKHART, S.H. et al. Depression of ventilation by desflurane in humans. Anesthesiology, Hagerstown, v.74, n.3, p.484488, 1991.

LOWE, D. et al. Influence of volatile anesthetics on left ventricular afterload in vivo. Differences between desflurane and sevoflurane. Anesthesiology, Hagerstown, v.85, n.1, p.112-120, 1996.

LUNA, S.P.L. Equilíbrio ácido-básico. In: FANTONI, D.T.; CORTOPASSI, S.R. Anestesia em cães e gatos. São Paulo: Roca, 2002. p.120-129.

McMURPHY, R.M.; HODGSON, D.S. Cardiopulmonary effects of desflurane in cats. American Journal of Veterinary Research, Schaumburg, v.57, n.3, p.367-370, 1996.

MORRISON, D.F. Multivariate statistical methods. New York: MaC Grows Hill Book, 1967. 388p.

NISHIMORI, C.T. Efeitos do óxido nitroso sobre parâmetros cardiovasculares, respiratórios e concentração alveolar mínima do desflurano, em cães. 2003. 87f. Dissertação (Mestrado em Cirurgia Veterinária)Faculdade de Ciências Agrárias e Veterinárias, Universidade Estadual Paulista, Jaboticabal.

NUNES, N. et al. Efeitos da associação de fentanil e droperidol na concentração alveolar mínima do desflurano, em cães. ARS Veterinária, Jaboticabal, v.17, n.2, p.86-91, 2001.

NUNES, N. et al. Effects of desflurane, sevoflurane and isoflurane on pulmonary shunt in dogs during spontaneous ventilation. Arquivo Brasileiro de Medicina Veterinária e Zootecnia, Belo Horizonte, v.55, n.2, p.173-177, 2003.

PACENTINE, G.G. et al. Effects of fentanyl on sympathetic activation associated with the administration of desflurane. Anesthesiology, Hagerstown, v.82, p.823-831, 1995.
PAGEL, P.S. et al. Desflurane and isoflurane produce similar alterations in systemic and pulmonary hemodynamic and arterial oxygenation in patient undergoing one-lung ventilation during thoracotomy. Anesthesia and Analgesia, New York, v.87, n.4, p.800-807, 1998

QUANDT, J.E. et al. Butorphanol does not reduce the minimum alveolar concentration of halothane in dogs. Veterinary Surgery, Philadelphia, v.23, n.2, p.156-159, 1994.

RODIG, G. et al. Effects of rapid increases of desflurane and sevoflurane to concentrations of 1.5 MAC on systemic vascular resistance and catecholamine response during cardiopulmonary bypass. Anesthesiology, Hagerstown, v.87, n.4, p.801-807, 1997.

SANTOS, P.S.P Efeitos da associação de fentanil e droperidol sobre a dinâmica cardiorrespiratória e concentração alveolar mínima do desflurano, em cães. 2000. 100f. Dissertação (Mestrado em Cirurgia Veterinária) Faculdade de Ciências Agrárias e Veterinárias, Universidade Estadual Paulista, Jaboticabal

SCRIVANI, P.V. et al. Effects of acepromazine and butorphanol on positive-contrast upper gastrointestinal tract examination in dogs. American Journal of Veterinary Research, Schaumburg, v.59, n.10, p.1227-1233, 1998.

SEBEL, P.S. et al. Reduction of the MAC of desflurane with fentanyl. Anesthesiology, Hagerstown, v.76, p.52-59, 1992.

THURMON, J.C. et al. Preanesthetics and anesthetic adjuncts. In: Lumb \& Jones' veterinary anesthesia. 3.ed. Philadelphia: Lea \& Feabiger, 1996. p.191-192.

TRIM, C.M. Cardiopulmonary effects of butorphanol tartrate in dogs. American Journal of Veterinary Research, Schaumburg, v.44, n.2, p.329-331, 1983.

TYNER, C.L. et al. Cardiovascular effects of butorphanol administration in isoflurane- $\mathrm{O}_{2}$ anesthetized healthy dogs. American Journal of Veterinary Research, Schaumburg, v.50, n.8, p.1340-1342, 1989.

VALVERDE, A. et al. Epidural morphine reduces halothane MAC in the dog. Canadian Journal of Anaesthesia, Ontário, v.36, n.6, p.629-632, 1989.

WATERMAN, A.E. et al. Analgesic activity and respiratory effects of butorphanol in sheep. Research in Veterinary Science, London, v.51, n.1, p.19-23, 1991.

WHITE, D.A. et al. Decrease in vascular resistance in the isolated canine hindlimb after graded doses of alfentanil, fentanyl, and sulfentanil. Anesthesia and Analgesia, New York, v.70, n.1, p.29-34, 1990.

ZORAN, D.L. et al. Pharmacokinetics of propofol in mixedbreed dogs and greyhounds. American Journal of Veterinary Research, Schaumburg, v.54, n.5, p.755-760, 1993. 